\title{
Relación entre conciencia ambiental y conducta pro ambiental en estudiantes de primer ciclo de la Universidad María Auxiliadora Lima-Perú
}

\author{
Relation between environmental awareness and pro environmental behavior in \\ first-cycle students at Maria Auxiliadora University Lima-Peru
}

\author{
${ }^{1}$ Victor Chero Pacheco \\ ${ }^{2}$ José Oruna Lara
}

\author{
${ }^{3}$ Silvia Jaimes Garay \\ ${ }^{4}$ Meri Tovar Baca
}

\section{RESUMEN}

El presente estudio tuvo como objetivo determinar la relación entre la conciencia ambiental y la conducta pro ambiental en los estudiantes de primer ciclo de la Universidad María Auxiliadora. De acuerdo a los materiales y métodos, fue un estudio con enfoque cuantitativo, diseño no experimental, relacional y transversal. La muestra estuvo constituida por 236 estudiantes seleccionados por muestreo probabilístico de una población de 610 estudiantes de primer ciclo. El instrumento de recolección de datos utilizado para la medición de la conciencia ambiental fue la Escala Nuevo Paradigma Ecológico Revisada conformada por 15 ítems, y el instrumento de recolección de datos utilizado para la medición de la conducta pro ambiental fue adaptado a partir de la Escala de Conductas Protectoras del Ambiente, conformada por 16 ítems. En relación a la conciencia ambiental, 223 estudiantes (94.5\%) muestran un nivel apropiado (alternativas "de acuerdo" y "completamente de acuerdo"), 13 estudiantes (5.5\%) presentan un nivel no apropiado (alternativas "completamente en desacuerdo", "en desacuerdo" y "no estoy seguro"). En relación a la conducta pro ambiental, 133 estudiantes (56.3\%) muestran una conducta apropiada (alternativas "muchas veces" "y "siempre") y 103 estudiantes (43.7\%) muestran una conducta no apropiada (alternativas "casi nunca" y "a veces"). Se concluye que, existe predominio de un nivel alto de conciencia ambiental, además predominio de una conducta pro ambiental apropiada. Existe relación directa entre la conciencia ambiental y la conducta pro ambiental.

Palabras clave: Conciencia ambiental, conducta pro ambiental, medio ambiente.

\begin{abstract}
The aim of this study was to determine the relation between environmental awareness and pro-environmental behavior of first-cycle students in Maria Auxiliadora University. According to materials and methods, it was a quantitative approach study and it was a non-experimental, correlational and transversal design. The sample included 236 students selected by probability sampling from 610 first-cycle students at Maria Auxiliadora University. It was used the Revised New Paradigm Environmental Scale that included 15 items to measure the environmental awareness, and the pro-environmental behavior was measured using a modified scale from the original Environmental Protection Behavior Scale that included 16 items. Regarding the environmental awareness, 223 students (94.5\%) students had an appropriate level (agree and completely agree options), and 13 students (5.5\%) had a low level (completely disagree, disagree and unsure options). In relation to pro-environmental behavior, 133 students $(56.3 \%$ ) developed an appropriate behavior (many times and always options), and 103 students $(43.7 \%)$ developed an inappropriate behavior (sometimes and hardly ever options). There was direct relation between environmental awareness and pro-environmental behavior. It was concluded that there was a high level of environmental awareness. Pro-environmental behavior was developed appropriately. There was direct relation between environmental awareness and pro-environmental behavior.
\end{abstract}

Keywords: Environmental awareness, pro-environmental behavior, environment. 


\section{INTRODUCCIÓN}

El ambiente universitario es propicio para la transmisión de información respecto al cuidado del medio ambiente. De acuerdo a esto, habría que considerar si la preocupación sobre las propiedades del entorno ambiental, se halla en relación a una conducta adecuada hacia la protección del mismo. Puede considerarse que, la instauración de un estado de preocupación por la conservación ambiental sería indispensable como un paso previo al desarrollo de estrategias dirigidas al control y recuperación de los elementos constituyentes del ambiente, pero dada la complejidad de la conducta humana, esta podría orientarse de acuerdo a diversos estímulos que no obedezcan necesariamente al aspecto reflexivo. El cuidado del medio ambiente inculcado a los estudiantes puede requerir el empleo de estrategias educativas que posibiliten la introducción de conceptos nuevos para su comprensión inicial y posterior toma de conciencia. Puede ser necesario, además, estimular la participación activa en tareas de prevención y control respecto a la afección del entorno. Es necesario resaltar las características del estudio en un ámbito específico y reducido, ya que situaciones como las referidas en la presente investigación más que la búsqueda de generalización de resultados, se orienta a la identificación exacta de elementos en un área reducida, desde donde es más precisa la adecuación o aplicación de estrategias para optimizar resultados.

Respecto a la conciencia, Dehaene (2015) y Valderas (2017) concuerdan en que involucra percepciones, pensamientos, sentimientos y sociabilidad; es decir, se trata de un proceso por el cual los individuos se percatan de los fenómenos. Asimismo, se considera que la conciencia es un saber implícito en algo; es decir, cuando nos hacemos conscientes, lo que hacemos es recuperar la conciencia de algo que ya existe (Scaramelli, 2011). Las características previamente consideradas serán aplicadas al identificar las alteraciones o daño ambiental pudiendo establecerse un diagnóstico o materia de preocupación, siendo esto el fundamento de lo que es en esencia la conciencia ambiental. Por su parte, Mosterín (2010) señala la importancia de la conciencia para identificar los desórdenes progresivos en el entorno del ser humano.

En relación a la conducta como eje guía de las actividades desarrolladas por los individuos, esta se manifiesta como una reacción de los sujetos frente a diversas situaciones (Consuegra, 2009). Además, dicha conducta puede observarse y medirse. De acuerdo a los factores que originan la conducta, los fenómenos psíquicos son aquellos que propician las manifestaciones corporales, lo cual es denominado perspectiva idealista; además, la influencia social también contribuye sobre la conducta, y esto es considerado como una concepción materialista (Grande-García, 2013).

$\mathrm{Al}$ desarrollar una conducta dirigida a proteger el entorno físico o natural se establece la definición de conducta pro ambiental o llamada también pro ecológica. Con base en las actividades específicas que dicha conducta incluye, son señalados el reciclaje, acciones de estética ambiental, compra de productos no dañinos para el ambiente, ahorro de agua, ahorro de energía eléctrica, reducción en el uso de automóviles, ahorro de combustible, revisión de temas ambientales, persuasión pro ecológica (Corral, 2012). El ser humano, gracias a su conducta, es capaz de controlar y atenuar los efectos nocivos sobre el ambiente, ya que se dispone de la tecnología necesaria e idónea para cada aspecto involucrado (Flannery, 2011; Williams, 2018; Lanegra, 2017).

El estudio se justifica por la importancia de la recuperación del entorno o mitigación de efectos dañinos a causa de la actividad humana, lo cual debe tomarse en cuenta en las instituciones educativas; en el presente caso, desde el ámbito universitario.

Considerando lo señalado, el objetivo principal del presente estudio consiste en determinar la relación entre la conciencia ambiental y la conducta pro ambiental en los estudiantes universitarios de primer ciclo.

\section{MATERIAL Y MÉTODOS}

El estudio es cuantitativo con diseño no experimental, relacional y transversal. Se ejecutó en el distrito de San Juan de Lurigancho, siendo el ámbito específico la Universidad María Auxiliadora. La población estuvo constituida por 236 estudiantes seleccionados por muestreo probabilístico de una población de 610 estudiantes de primer ciclo de las facultades de Ciencias de la Salud y Ciencias Empresariales. Los criterios de inclusión fueron: Estudiantes regulares de la universidad, pertenecientes al primer ciclo de estudios. Se excluyeron a aquellos que por diversas circunstancias presentaban dificultades para el normal desarrollo de sus 
actividades en la institución. La técnica de recolección de datos fue la encuesta y los instrumentos empleados correspondieron a escalas. Se aplicaron dichos instrumentos en las aulas.

Para evaluar la conciencia ambiental, se empleó la Escala Nuevo Paradigma Ecológico Revisada desarrollada por Dunlap, Van Liere, Mertig y Jones (2000) (Adaptación de la escala NEP del año 1978 elaborada por Dunlap y Van Liere) (Dunlap \& Van Liere, 2008). La medición de la conducta pro ambiental se realizó por medio de un instrumento adaptado a partir de la Escala de Conductas Protectoras del Ambiente de Corral, Hess, Hernández y Suárez elaborada en el año 2002 (Corral, Frías y González, 2003).

Para el análisis de datos se empleó el programa estadístico IBM SPSS versión 23, desarrollando el análisis descriptivo de las dimensiones $e$ indicadores correspondientes, así como el análisis inferencial durante la evaluación de la relación (prueba de correlación Rho de Spearman) entre variables. De acuerdo al aspecto ético, las actividades desarrolladas en el estudio no representaron riesgo alguno para los individuos participantes quienes aceptaron voluntariamente su inclusión tras la comunicación de las características y procedimientos involucrados.

\section{RESULTADOS}

En la tabla 1 se evidencia el predominio de la opción "Completamente de acuerdo" con 137 casos (58.1\%), seguido de las opciones "De acuerdo", "No estoy seguro", "En desacuerdo" y "Completamente en desacuerdo con 86 casos (36.4\%), 9 casos (3.8\%), 3 casos $(1.3 \%)$ y 1 caso $(0.4 \%)$, respectivamente. Considerando que las respuestas favorables corresponden a las opciones "Completamente de acuerdo" y "De acuerdo", se asume que es apropiado el nivel de conciencia ambiental.

Tabla1. Conciencia ambiental en estudiantes de primer ciclo de la Universidad María Auxiliadora

\begin{tabular}{|llcccc|} 
& Frecuencia & Porcentaje & $\begin{array}{c}\text { Porcentaje } \\
\text { válido }\end{array}$ & $\begin{array}{r}\text { Porcentaje } \\
\text { acumulado }\end{array}$ \\
\hline \multirow{3}{*}{ Válidos } & Completamente en desacuerdo & 1 & 0.4 & 0.4 & 0.4 \\
& En desacuerdo & 3 & 1.3 & 1.3 & 1.7 \\
& No estoy seguro & 9 & 3.8 & 3.8 & 5.5 \\
& De acuerdo & 86 & 36.4 & 36.4 & 41.9 \\
& Completamente de acuerdo & 137 & 58.1 & 58.1 & 100 \\
& Total & 236 & 100 & 100 &
\end{tabular}

En la tabla 2 se evidencia el predominio de la opción "Muchas veces" con 115 casos (48.7\%), seguido de la opción "A veces" con 95 casos (40.3\%). El tercer lugar corresponde a la opción "Siempre" con 18 casos $(7.6$ $\%)$.
Finalmente, el cuarto lugar corresponde a la opción "Casi nunca" con 8 casos (3.4\%). Considerando que las respuestas favorables corresponden a las opciones "Muchas veces" y "Siempre", se asume que la conducta pro ambiental es desarrollada apropiadamente.

Tabla 2. Conducta pro ambiental en estudiantes de primer ciclo de la Universidad María Auxiliadora

\begin{tabular}{|c|c|c|c|c|c|}
\hline & & Frecuencia & Porcentaje & $\begin{array}{c}\text { Porcentaje } \\
\text { válido }\end{array}$ & $\begin{array}{l}\text { Porcentaje } \\
\text { acumulado }\end{array}$ \\
\hline \multirow{5}{*}{ Válidos } & Casi nunca & 8 & 3.4 & 3.4 & 3.4 \\
\hline & A veces & 95 & 40.3 & 40.3 & 43.6 \\
\hline & Muchas veces & 115 & 48.7 & 48.7 & 92.4 \\
\hline & Siempre & 18 & 7.6 & 7.6 & 100 \\
\hline & Total & 236 & 100 & 100 & \\
\hline
\end{tabular}


En la tabla 3, se evidencia que el resultado de la prueba no paramétrica Rho de Spearman es 0.210, lo cual sugiere una relación no muy significativa pero positiva. El valor en la prueba de significación bilateral de 0.001 resulta menor a la probabilidad de 0.05 , lo cual corrobora la existencia de relación directa entre las variables.

Tabla 3. Relación entre la conciencia ambiental y la conducta pro ambiental

\begin{tabular}{|lllcc} 
& & & $\begin{array}{c}\text { Conciencia } \\
\text { ambiental }\end{array}$ & $\begin{array}{c}\text { Conducta pro } \\
\text { ambiental }\end{array}$ \\
\hline & Conciencia & Coeficiente de correlación & 1.000 & 0.210 \\
Rho de & ambiental & Sig. (bilateral) & & 0.001 \\
Spearman & N & Coeficiente de correlación & 0.210 & 236 \\
& Conducta pro & Sig. (bilateral) & 0.001 & 1.000 \\
& ambiental & N & 236 & 236 \\
\end{tabular}

En la tabla 4 se evidencia, de acuerdo al valor de la significancia inferior a $0.05(5 \%)$, relación entre los resultados de los ítems p2:c11, p2:c16, p3:c6, p3:c13, p3:c14, p5:c8, p5:c9, p5:c10, p5:c11, p5:c12, p5:c13, p5:c14, p7:c12, p8:c12, p9:c12, p10:c11, p10:c12, p10:c14, p10:c15, p11:c3, p11:c11, p11:c13, p11:c15, p12:c1, p12:c3, p12:c12, p12:c14, p13:c3, p13:c6, p13:c7, p13:c11, p13:c12, p13:c14, p13:c16, siendo señaladas las Escalas con los ítems respectivos.

Escala Nuevo Paradigma Ecológico Revisada (Dunlap, Van Liere, Mertig y Jones, 2000)

1. Nos estamos aproximando al número límite de personas que la tierra puede albergar.

2 . Los seres humanos no tienen derecho a modificar el medio ambiente para adaptarlo a sus necesidades.

3. Cuando los seres humanos interfieren en la naturaleza, a menudo las consecuencias son desastrosas.

4. La creatividad humana no evitará que el planeta se dañe.

5. Los seres humanos están abusando seriamente del medio ambiente.

6. La tierra tiene recursos naturales que deben ser protegidos.

7. Las plantas y los animales tienen tanto derecho como los seres humanos a existir.

8. La naturaleza no puede soportar todo el daño que le causan.

9. Los seres humanos debemos respetar las leyes de la naturaleza.

10. La humanidad será afectada por los problemas ambientales.
11. La tierra tiene recursos que pueden agotarse.

12. Los seres humanos no tienen derecho a dominar la naturaleza.

13. El equilibrio de la naturaleza es muy delicado y fácilmente alterable.

14. Los seres humanos no han cuidado adecuadamente a la naturaleza.

15. Si las cosas continúan como hasta ahora, pronto experimentaremos una gran catástrofe ecológica.

Escala de Conductas Protectoras del Ambiente (Corral, Frías y González, 2009).

1. Leo sobre temas de Ecología.

2. Llamo la atención a quien daña el ambiente.

3. Converso sobre temas de Ecología.

4. Veo o escucho programas sobre Ecología.

5. Recojo envolturas o envases descartables que otras personas arrojan.

6. Apago las luces si otros las dejan encendidas.

7. Apago la radio o la televisión si otros las dejan encendidas.

8. Al lavar utensilios cuido el agua.

9. Al lavar ropa cuido el agua.

10. Al cepillarme los dientes cuido el agua.

11. Uso los frascos de vidrio de los productos envasados que he comprado.

12. Cuando escribo sobre papel trato de abarcar la mayor superficie posible.

13. Uso las latas de aluminio de los productos envasados que he comprado.

14. Compro productos elaborados con materiales reciclados.

15. Compro productos que no dañen el ambiente.

16. Compro bebidas con envase retornable. 
Tabla 3. Relación entre la conciencia ambiental y la conducta pro ambiental

\begin{tabular}{|c|c|c|c|c|c|c|c|c|c|c|c|c|c|c|c|c|c|}
\hline & & c1 & c2 & c3 & c4 & c5 & c6 & c7c & c8 & c9 & c10 & c11 & c12 & c13 & c14 & c15 & c16 \\
\hline \multirow{3}{*}{$\mathrm{p} 1$} & Corr. & 0.052 & 0.014 & 0.113 & 0.040 & -0.076 & 0.034 & 0.075 & -0.101 & -0.046 & -0.034 & 0.024 & 0.043 & 0.099 & 0.098 & -0.007 & 0.008 \\
\hline & & & & & & & & & & & & & & & & & \\
\hline & Sig. & 0.428 & 0.834 & 0.084 & 0.537 & 0.245 & 0.608 & 0.251 & 0.122 & 0.481 & 0.601 & 0.715 & 0.514 & 0.131 & 0.132 & 0.914 & 0.901 \\
\hline \multirow{2}{*}{ p2 } & Corr. & 0122 & 0.031 & 0.104 & 0.124 & -0.106 & 0.024 & 0.035 & 0.015 & -0.030 & 0.006 & $0.144^{*}$ & 0.102 & 0.043 & 0.064 & 0.071 & $0.143^{\circ}$ \\
\hline & Sig. & 0.062 & 0.640 & 0.112 & 0.057 & 0.104 & 0.718 & 0.595 & 0.821 & 0.647 & 0.926 & 0.027 & 0.117 & 0.515 & 0.327 & 0.281 & 0.028 \\
\hline \multirow{2}{*}{ p3 } & Corr. & 0.101 & -0. & 0.072 & 0.096 & -0.007 & 0.169 & 0.101 & 0.081 & 0.038 & -0.003 & 0.090 & 0.068 & 0.137 & $0.162^{\circ}$ & 0.058 & 0.057 \\
\hline & Sig. & 0.121 & 0.773 & 0.272 & 0.140 & 0.912 & 0.009 & 0.121 & 0.215 & 0.559 & 0.967 & 0.168 & 0.298 & 0.035 & 0.013 & 0.372 & 0.383 \\
\hline \multirow{3}{*}{ p4 } & Corr. & 0.028 & 0.068 & 0.015 & 0.007 & -0.013 & 0.016 & 0.084 & 0.009 & 0.062 & 0.114 & 0.010 & -0.019 & -0.026 & -0.015 & 0.086 & 0.125 \\
\hline & & & & & & & & & & & & & & & & & \\
\hline & Sig. & 0.672 & 0.302 & 0.813 & 0.911 & 0.849 & 0.807 & 0.198 & 0.890 & 0.343 & 0.080 & 0.879 & 0.771 & 0.686 & 0.820 & 0.187 & 0.054 \\
\hline \multirow{2}{*}{ p5 } & Corr. & -0.030 & 0.058 & 0.048 & 0.097 & 0.009 & 0.106 & 0.055 & $0.140^{*}$ & $0.128^{*}$ & 0.179 & $0.130^{*}$ & $0.177^{*+*}$ & 0.141 & $0.134^{*}$ & 0.101 & 0.024 \\
\hline & Sig. & 0.646 & 0.374 & 0.461 & 0.139 & 0.885 & 0.105 & 0.404 & 0.032 & 0.049 & 0.006 & 0.046 & 0.006 & 0.030 & 0.040 & 0.122 & 0.709 \\
\hline \multirow{2}{*}{ p6 } & Corr. & -0.029 & -0. & 0.061 & 0.024 & -0.002 & 0.098 & 0.034 & 0.066 & -0.006 & 0.041 & 0.055 & 0.098 & 0.088 & 0.114 & 0.110 & 0.046 \\
\hline & Sig. & 0.662 & 0.915 & 0.351 & 0.713 & 0.978 & 0.132 & 0.606 & 0.313 & 0.926 & 0.531 & 0.399 & 0.135 & 0.175 & 0.081 & 0.091 & 0.480 \\
\hline \multirow{2}{*}{ p7 } & Corr. & -0.030 & -0 . & -0.052 & 0.007 & 0.032 & -0.005 & 0.024 & 0.057 & -0.029 & 0.017 & 0.084 & $0.128^{*}$ & 0.092 & 0.062 & 0.020 & -0.012 \\
\hline & Sig. & 0.642 & 0.317 & 0.424 & 0.912 & 0.630 & 0.940 & 0.718 & 0.383 & 0.657 & 0.794 & 0.199 & 0.049 & 0.160 & 0.346 & 0.765 & 0.857 \\
\hline \multirow{2}{*}{ p8 } & Corr. & -0.046 & 0.009 & 0.089 & 0.044 & -0.071 & 0.058 & 0.047 & 0.031 & 0.041 & 0.020 & 0.126 & $0.150^{*}$ & 0.123 & 0.120 & 0.090 & 0.058 \\
\hline & Sig. & 0.480 & 0.890 & 0.173 & 0.501 & 0.280 & 0.376 & 0.474 & 0.638 & 0.533 & 0.762 & 0.054 & 0.021 & 0.060 & 0.066 & 0.170 & 0.379 \\
\hline \multirow{2}{*}{ p9 } & Corr. & -0.019 & 0.001 & 0.010 & 0.004 & -0.005 & 0.020 & 0.032 & 0.054 & 0.048 & 0.023 & 0,105 & $0.159^{*}$ & 0.079 & 0.077 & 0.061 & 0.031 \\
\hline & Sig. & 0.775 & 0.984 & 0.880 & 0.956 & 0.939 & 0.755 & 0.621 & 0.407 & 0.466 & 0.728 & 0.108 & 0.014 & 0.225 & 0.237 & 0.349 & 0.632 \\
\hline \multirow{2}{*}{10} & Corr. & 0.057 & 0.068 & $0.155^{\circ}$ & 0.051 & 0.061 & 0.124 & 0.111 & 0.071 & 0.037 & 0.110 & $0.227^{* *}$ & $0.215^{\text {\#* }}$ & 0.085 & $0.135^{*}$ & $0.216^{* *}$ & $0.128^{\circ}$ \\
\hline & Sig. & 0.381 & 0.295 & 0.017 & 0.439 & 0.353 & 0.058 & 0.088 & 0.277 & 0.569 & 0.092 & 0.000 & 0.001 & 0.193 & 0.039 & 0.001 & 0.050 \\
\hline \multirow{2}{*}{ p11 } & Corr. & 0.079 & 0.021 & 0.143 & 0.057 & -0.001 & 0.023 & 0.049 & 0.087 & 0.037 & 0.034 & $0.213^{* *}$ & 0.120 & 0.144 & 0.126 & $0.231^{\text {** }}$ & 0.029 \\
\hline & Sig. & 0.224 & 0.751 & 0.028 & 0.385 & 0.994 & 0.728 & 0.451 & 0.183 & 0.568 & 0.602 & 0.001 & 0.065 & 0.027 & 0.053 & 0.000 & 0.656 \\
\hline \multirow{3}{*}{ p12 } & Corr. & $0.129^{*}$ & -0 & 0.172 & 0.082 & -0.039 & 0.018 & 0.035 & 0.056 & 0.015 & 0.048 & 0.124 & $0.192^{* * *}$ & 0.078 & $0.142^{\circ}$ & 0.101 & 0.022 \\
\hline & & & & & & & & & & & & & & & & & \\
\hline & Sig. & 0.048 & 0.907 & 0.008 & 0.211 & 0.546 & 0.783 & 0.589 & 0.395 & 0.816 & 0.464 & 0.057 & 0.003 & 0.232 & 0.029 & 0.124 & 0.737 \\
\hline \multirow{3}{*}{ p13 } & Corr. & 0.101 & 0.048 & 0.159 & 0.062 & -0.043 & 0.161 & $0.133^{*}$ & 0.052 & 0.033 & 0.055 & $0.169^{* *}$ & $0.188^{* *}$ & 0.111 & $0.159^{*}$ & 0.081 & $0.140^{*}$ \\
\hline & & & & & & & & & & & & & & & & & \\
\hline & Sig. & 0.122 & 0.466 & 0.014 & 0.339 & 0.515 & 0.013 & 0.042 & 0.424 & 0.609 & 0.400 & 0.009 & 0004 & 0.088 & 0.014 & 0.214 & 0.032 \\
\hline \multirow{3}{*}{ p14 } & Corr. & 0029 & 0.049 & 0.102 & 0.048 & 0.002 & 0.044 & 0.114 & 0.053 & 0.040 & 0.045 & 0.075 & 0.099 & 0.083 & 0.076 & 0.063 & 0.020 \\
\hline & & & & & & & & & & & & & & & & & \\
\hline & Sig. & 0.657 & 0.456 & 0.118 & 0.459 & 0.974 & 0.500 & 0.080 & 0.421 & 0.545 & 0.488 & 0.251 & 0.130 & 0.205 & 0.244 & 0.339 & 0.759 \\
\hline \multirow{3}{*}{ p15 } & Corr. & -0.061 & 0.007 & 0.074 & 0.051 & 0.088 & 0.061 & 0.078 & 0.023 & -0.005 & -0.007 & 0.071 & 0.061 & 0.000 & 0.009 & 0.021 & 0.049 \\
\hline & & & & & & & & & & & & & & & & & \\
\hline & Sig. & 0.351 & 0.918 & 0.261 & 0.439 & 0.178 & 0.350 & 0.232 & 0.729 & 0.939 & 0.921 & 0.276 & 0.352 & 0.994 & 0.896 & 0.745 & 0.456 \\
\hline
\end{tabular}

\section{DISCUSIÓN}

Es indispensable mantener el equilibrio en el entorno o medio ambiente; es decir, cada uno de los elementos que lo conforman debe complementarse con los demás, de acuerdo a las propiedades intrínsecas que posibilitan el carácter integrador. Si dicha integración se presenta de forma ordenada, constante o cíclica, los resultados se evidenciarán por un entorno favorable en donde los seres vivos desarrollan y conviven gracias a las posibilidades de sustento brindadas por las propias riquezas del espacio ambiental. Lamentablemente, los espacios o más específicamente determinados elementos o recursos han sufrido deterioro y dicho estado, aunque parezca contradictorio, ha resultado de la actividad de quienes por siglos se vieron 
beneficiados con las bondades brindadas por la naturaleza. En otras palabras, la actividad del ser humano que por descuido o en algunos casos, ambición, ha empleado su dominio para sustraer exageradamente elementos que difícilmente se recuperan.

A pesar de las dificultades para recuperar íntegramente el entorno, deben desarrollarse estrategias que eviten el progreso del daño ocasionado. Por tal motivo, es imprescindible el conocimiento y la inquietud por identificar aspectos nocivos para su control o mitigación.

En el presente estudio, la identificación de los aspectos dañinos señalados es considerada bajo el concepto de conciencia ambiental, en donde las alteraciones o eventos desfavorables deben ser identificados por los estudiantes en una primera instancia, determinando así aquello que es materia de preocupación para la búsqueda posterior de soluciones.

Los resultados respecto al nivel de conciencia ambiental se evidencian favorables en el grupo evaluado, siendo así capaces de analizar situaciones desfavorables, lo cual es un paso importante ya que no perciben como ajenas tales situaciones. Aminrad, Sayed, Samad \& Sakari (2013) identificaron, de forma similar, un nivel apropiado de conciencia ambiental en una población de estudiantes de nivel secundario al igual que Dicle, Isil \& Safiye (2010), quienes estudiaron dicha característica en una población de estudiantes universitarios. Es señalada, además, la preocupación por factores que de forma importante influyen sobre la conciencia ambiental. Así lo explican, Urda y Bonan (2016). El desarrollo de la conciencia ambiental pareciera ser importante para desencadenar conductas protectoras del entorno; es así que los resultados son consistentes con dicho enunciado, ya que la conducta resultante tras la medición estuvo representada por valores importantes. En concordancia a dichos resultados, Tien-Ming \& Homer (2015) señalaron un apropiado grado de conductas favorables hacia el cuidado ambiental, del mismo modo que Palavecinos, Amérigo, Ulloa y Muñoz (2016) determinaron en poblaciones de Sudamérica y Europa. En diversos estudios se toman en cuenta numerosos factores asociados a la conducta, por tal motivo resulta complejo hallar un solo parámetro que permita la orientación de la misma.

Los resultados en la presente investigación permiten establecer relación directa entre la conciencia ambiental y la conducta pro ambiental, lo cual es coherente con los resultados presentados por Palavecinos et al., (2016), quienes señalan la relación directa entre ambas variables, especificando además ciertas características de las poblaciones involucradas en su análisis. Otros elementos involucrados podrían influenciar en dichos resultados, lo cual da luces para incrementar estudios al respecto. La complejidad señalada anteriormente, respecto al estudio de la conducta se manifiesta en diversos estudios con resultados variables, difíciles de uniformizar o generalizar hacia todas las poblaciones. Tonelo y Valladares (2015) presentan también resultados que permiten establecer una relación directa e importante entre conciencia ambiental y conducta sustentable. Las creencias y diversas normas de carácter subjetivo también pueden ser consideradas como influyentes en el desarrollo de la conducta protectora hacia el ambiente (Torres, Barreto y Vásquez, 2015).

Las características propias de los diversos estudios requieren la consideración o desarrollo de investigaciones constantes incluyendo múltiples variables, ya que cada uno de los aspectos específicos servirá para incrementar el conocimiento sobre la complejidad e importancia de la conducta como consecuencia fundamental con carácter significativo modificador de las particularidades del medio o entorno ambiental.

\section{CONCLUSIONES}

Existe predominio de un nivel alto de conciencia ambiental representado por las opiniones favorables al identificar las características de deterioro ambiental y reconociendo la responsabilidad de los seres humanos. Existe predominio de una conducta pro ambiental apropiada, al identificar un nivel elevado de conductas que se orientan a la recuperación o mitigación de eventos adversos. En el presente estudio se evidencia relación directa entre la conciencia ambiental y la conducta pro ambiental, por lo que el grado de preocupación respecto al daño del ambiente se puede reflejar en las conductas protectoras del entorno. A pesar de considerar herramientas que de manera subjetiva evalúan las variables de estudio, siendo esto una posible limitante para el análisis de resultados, debe considerarse que la cuantificación aplicada proporciona parámetros de orientación para desarrollar estudios desde otros enfoques, habiendo sido algunos señalados en estudios previos. 


\section{REFERENCIAS BIBLIOGRÁFICAS}

Aminrad, Z., Sayed, S., Samad, A. \& Sakari, M. (2013). Relationship between awareness, knowledge and attitudes towards environmental education among secondary school students in Malaysia. World Applied Sciences Journal, 22(9), 1326-1333. Recuperado de https://www.research gate.net/publication/286958008_Rela tionship_between_awareness_knowl edge and attitudes towards environ mental_education_among_secondary school_students_in_Malaysia

Consuegra, N. (2009). Diccionario de psicología. Recuperado de https://ebookcentral. proquest.com

Corral, V. (2012). Sustentabilidad y psicología positiva: una visión optimista de las conductas proambientales y prosociales. Recuperado de https://ebookcentral.proquest.com

Corral, V., Frías, M. \& González, D. (2003) Percepción de riesgos, conducta proambiental y variables demográficas en una comunidad de Sonora, México. Región y sociedad, 15(26), 49-72. Recuperado de http://www.scielo.org.mx/scielo.p $\mathrm{hp}$ ? script $=$ sci arttext\&pid $=\mathrm{S} 1870$ $39252003000100002 \& \operatorname{lng}=\mathrm{es} \& \operatorname{tlng}=$

Dehaene, S. (2015). La conciencia en el cerebro: descifrando el enigma de cómo el cerebro elabora nuestros pensamientos. Recuperado de https://ebookcentral.proquest.com

Dicle, O., Isil, C. \& Safiye, K. (2010). Environmental awareness of University Students in Ankara, Turkey. African Journal of Agricultural Research, 5(19), 26292636 . R e c u p e r a d o d e https://academicjournals.org/article/a rticle1380961160_Oguz\%20et\%20al. pdf

Dunlap, R. \& Van Liere, K. (2008). The "New Environmental Paradigm", The Journal of Environmental Education, 40(1), 19-28. Recuperado de https://www.researchgate.net/publica tion/254345132_The_New_Environ mental_Paradigm

Dunlap, R., Van Liere, K., Mertig, A. \& Jones, R.
(2000). Measuring Endorsement of the New Ecological Paradigm; A revised NEP Scale. Journal of Social Issues 56(3), 425-442. Recuperado de https://web.standford.edu/kcarmel /CC_BehavChange_course/readings/ Additional\%20Resources/J\%20Soc $\% 20 I s s u e s \% 202000 /$ dunlap_2000_5 nep_a.pdf

Flannery, $\overline{\mathrm{T}}$. (2011). El clima está en nuestras manos: Historia del calentamiento global. Lima, Perú: Taurus.

Grande-García, I. (2013). Psicología: historia, teoría y procesos básicos. Recuperado dehttps://ebookcentral.proquest.com

Lanegra, I. (2017). ¿Qué es el cambio climático? Lima, Perú: Editorial Planeta Perú S.A.

Mosterín, J. (2010). Naturaleza, vida y cultura. Lima, Perú: Fondo editorial de la Universidad Inca Garcilaso de la Vega.

Palavecinos, M., Amérigo, M., Ulloa, J. y Muñoz, J. (2016). Preocupación y conducta ecológica responsable en estudiantes universitarios: estudio comparativo entre estudiantes chilenos y españoles. Psychosocial Intervention, 25(3), 143-148. Recuperado de http://scielo.isciii.es/scielo.php?script =sci arttext\&pid=S1132-

Scaramelli, A. (2011). Evolución de la conciencia: dónde estoy y hacia dónde $\begin{array}{llll}v & y\end{array}$. $\mathrm{R}$ e c u p e $\mathrm{r}$ a d o $\mathrm{d} \mathrm{e}$ https://ebookcentral.proquest.com

Tien-Ming, Ch. \& Homer, C. (2015). How do environmental knowledge, environmental sensitivity, and place attachment affect environmentally responsible behavior? An integrated approach for sustainable island tourism, Journal of Sustainable Tourism, 23(4), 557-576. Recuperado de https://www.tandfonline.com/doi/ abs/10.1080/09669582.2014.965177

Tonello, G. y Valladares, N. (2015). Conciencia ambiental y conducta sustentable relacionada con el uso de energía para iluminación. Gestión y Ambiente, 18(1),45-59. Recuperado de http://www.redalyc.org/articulo.oa?id $=169439782003$

Torres, T., Barreto, I. y Vásquez, J. (2015). Creencias y normas subjetivas como predictores de intención de 
comportamiento proambiental. Suma psicológica, 22(2), 86-92. Recuperado de https //www.science direct.com/science/article/pii/S01214 38115000235

Urda, E. \& Bonan, L. (2016). Saber no alcanza para actuar: revisión y reflexiones acerca de la relación entre el conocimiento y la adopción de conductas ambientales. Ciência \& Educação (Bauru), 23(2), 357-372.
Recuperado de http://www.scielo.br/ s c i e 1 o.p h p ? p id = S 1516 $73132017000200357 \&$ script $=$ sci_abs tract\&tlng $=\mathrm{es}$

Valderas, J. (2017). La Conciencia. México, México: RBA Editores México.

Williams, F. (2018). La dosis natural. (1 $\left.1^{\mathrm{a}} \mathrm{ed}.\right)$. Bogotá, Colombia: Editorial Planeta Colombiana S.A. 